\title{
Comparison of different miR-21 inhibitor chemistries in a cardiac disease model
}

$W_{0}$ that analyzed the role of microRNA-21 (miR-21) in a mouse model of cardiac disease (1). Using miR-21-deficient mice and novel, very short, 8-nucleotide anti-miR21 oligonucleotides, the authors failed to detect any modulation of pressure overload-induced myocardial hypertrophy and fibrosis and concluded that miR-21 plays no role in cardiac disease. In contrast, we and others reported that inhibition of miR-21 with highly specific, 22- and 15nucleotide-long anti-miR-21 oligonucleotides effectively inhibits myocardial and pulmonary fibrosis $(2,3)$. While genetic deletion of a target may lead to compensation during development and is often different from pharmacological inhibition of this target in the adult organism, the discrepancy between the therapeutic trials using long versus short 8-mer oligonucleo- tides is striking. We therefore carried out a direct head-to-head comparison of three different oligonucleotide chemistries (Figure 1A) in the same model of pressure overload-induced cardiac hypertrophy (transaortic constriction [TAC]). The two 22-mer oligonucleotides were complementary to the full-length miR-21, while the 8-mer was complementary to nucleotides 2 to 9 of miR-21, locked nucleic acid modified (LNA modified), and identical to the oligonucleotide used in the report by Patrick et al. (Figure 1A).

Therapentic efficacy of different anti-miR-21s in a mouse model of cardiac disease. Consistent with the results by Patrick et al., 8-mer antimiR-21 led to repression of cardiac miR21 on day 2 after the last dose (Figure 1B). However, treatment with 22-mer anti-miR$21 \mathrm{~s}$ resulted in a more efficacious repression of miR-21 (by $80 \%$ ). In addition, the repres- sion was maintained only in samples treated with 22-mers but not 8-mer throughout the course of the experiment (on day 19 after the last dose, Figure 1B). It is well established that the number of phosphothioate bonds is inversely correlated to the excretion rate of oligonucleotides (4), which could partially explain the lack of efficacy of 8-mer anti-miR-21 in the cardiac fibrosis model. In addition to comparing the ability of three different anti-miR-21 oligonucleotides to repress miR-21, we also tested their ability to modify disease phenotypes of TAC. As shown in Figure 1C, interstitial fibrosis and cardiac mass were significantly increased three weeks after TAC in control mice but were strongly attenuated by treatment with both cholesterol- and F/MOE-modified long 22-mer oligonucleotides. In addition, 22mers prevented the decline in cardiac function, as determined by echocardiography. In

\section{A}

22-mer Chol 5'-UCAACAUCAGUCUGAUAAGCUA-3 22-mer FMOE 5'-TCAACAUCAGUCTGAUAAGCTA-3' 8-mer 5'-GATAAGCT-3

2'-O-Methyl 2'Fluoro 2'-O-MethoxyEthyl Locked Nucleic Acid (LNA)

\section{B}

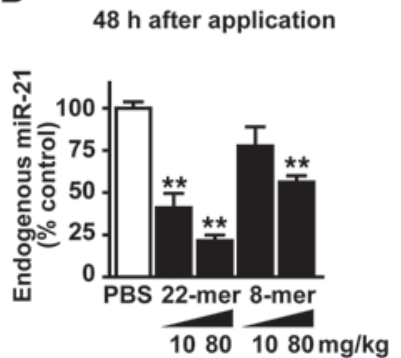

Day 19 after application

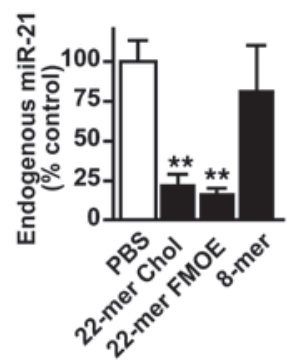

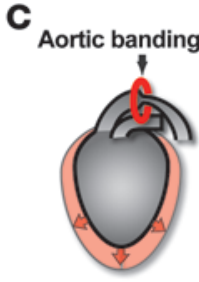

Aortic i.v. Ant-21 banding 8 -mer/22-mer $t^{\# 1+2}$

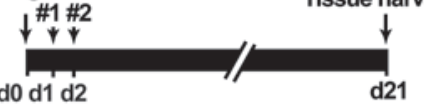

d0 d1 d2
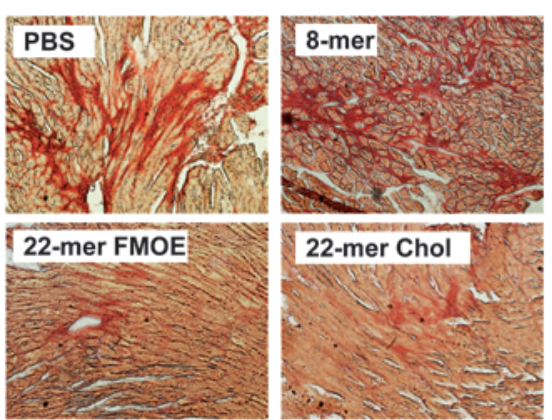
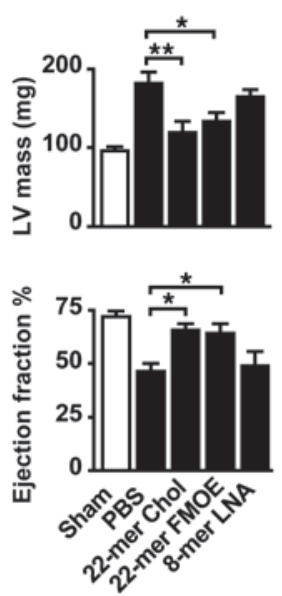

\section{Figure 1}

Comparison of different miR-21 oligonucleotide inhibitors. (A) Different chemistries of miR-21 inhibitors (modifications of oligonucleotides are indicated with corresponding colors in the key). (B) miR-21 expression 48 hours and 19 days after treatment (21 days after TAC) with different miR-21 inhibitors (10-80 mg/kg). (C) Efficacy of different miR-21 inhibitors in a mouse model of left ventricular pressure overload (TAC) on left ventricular fibrosis formation, left ventricular mass, and ejection fraction. The different miR-21 oligonucleotide inhibitors have been applied twice on two consecutive days (\#1 and \#2). Original magnification, $\times 200$. Ant-21, anti-miR-21; Chol, cholesterol. Data are mean \pm SEM. ${ }^{\star} P<0.05,{ }^{* *} P<0.01$. 
contrast and consistent with the findings reported by Patrick et al., application of short 8-mer oligonucleotides against miR21 did not affect pressure overload-induced cardiac hypertrophy, fibrosis, and cardiac dysfunction. Currently, we do not know why the reported phenotype of the miR-21-deficient mice differs from that of mice that received treatment with long miR-21 inhibitors. Possible reasons are various means of genetic compensation upon constitutive deletion of the Mir21 gene as well as potential off-target effects of anti-miRs that evade current analysis.

Taken together, we confirmed that the 8-mer anti-miR-21 is ineffective in preventing cardiac disease in a mouse model of left ventricular pressure overload, a finding which is likely due to the modest and transient nature of miR-21 suppression by 8 -mers. For long-term inhibition of miR21 function in vivo, interventions based on longer anti-miRs are likely to prove superior, due to their high potency and treatment duration. In contrast, short 8-mer LNAmodified oligonucleotides against miR-21 are of less potency and without therapeutic effects in vivo.

\section{Acknowledgments}

We kindly acknowledge the support of the Deutsche Forschungsgemeinschaft (DFG TH903/10-1), the BMBF (01EO0802 and 01KU0902A), and the Fondation Leducq.

\section{Thomas Thum, ${ }^{1,2}$ Nelson Chau, ${ }^{3}$ Balkrishen Bhat, ${ }^{3}$ Shashi Kumar Gupta, ${ }^{1}$ Peter S. Linsley, ${ }^{3}$ Johann Bauersachs, ${ }^{2}$ and Stefan Engelhardt ${ }^{4}$}

${ }^{1}$ Institute for Molecular and Translational Therapeutic Strategies, IFB-Tx, and ${ }^{2}$ Department of Cardiology and Angiology, Hannover Medical School, Hannover, Germany. ${ }^{3}$ Regulus Therapeutics, San Diego, California, USA. ${ }^{4}$ Institute of Pharmacology and Toxicology, Technische Universitaet Muenchen, Munich, Germany.

Conflict of interest: Thomas Thum, Johann Bauersachs, and Stefan Engelhardt have filed a patent application for the use of miR21 and have received royalty fees through the University of Wuerzburg. Nelson Chau, Balkrishen Bhat, and Peter S. Linsley are employees of Regulus.
Address correspondence to: Thomas Thum, Hannover Medical School, Institute for Molecular and Translational Therapeutic Strategies, Carl-Neuberg-Str. 1, 30625 Hannover, Germany. Phone: 49.511.532.5272; Fax: 49.511.532.5274; E-mail: Thum. Thomas@mh-hannover.de. Or to: Stefan Engelhardt, Institute of Pharmacology and Toxicology, Technische Universitaet Muenchen, Munich, Germany. Phone: 49.89.4140.3260; Fax: 49.89.4140.3261; E-mail: stefan.engelhardt@tum.de.

J Clin Invest. 2011;121(2):461-462. doi:10.1172/JCI45938.

1. Patrick DM, et al. Stress-dependent cardiac remodeling occurs in the absence of microRNA-21 in mice. J Clin Invest. 2010;120(11):3912-3916.

2. Thum T, et al. MicroRNA-21 contributes to myocardial disease by stimulating MAP kinase signalling in fibroblasts. Nature. 2008; 456(7224):980-984.

3. Liu G, et al. miR-21 mediates fibrogenic activation of pulmonary fibroblasts and lung fibrosis. J Exp Med. 2010;207(8):1589-1597.

4. Levin AA, et al. Basic principles of the pharmacokinetics of antisense oligonucleotide drugs. In: Crooke ST, ed. Antisense Drug Technology: Principles, Strategies, and Applications. 2nd ed. Boca Raton, Florida, USA: CRC Press; 2007:183-215.

\section{Response to Thum et al.}

$T$ hum et al. conclude that microRNA-21 (miR-21) is essential for cardiac hypertrophy and fibrosis in response to pressure overload (1). They also claim that our failure to observe a blockade to these processes in mice treated with an 8 -mer locked nucleic acid-modified oligonucleotide against miR-21 (called Anti-21) (2) is due to the ineffectiveness of such inhibitors. We wish to point out several caveats to their study regarding the role of miR-21 in cardiac hypertrophy and their conclusions regarding the efficacy of the Anti-21 oligonucleotide.

First, we find that Anti-21 inhibits miR21 with a half-maximal inhibitory concentration of $0.9 \mathrm{nM}$, indicating the efficacy of Anti-21. Second, Thum et al. do not state the method they used to measure miR21 inhibition, though we assume it to be quantitative PCR (qPCR). In our hands, qPCR alone is unreliable for measuring miRNA inhibition, especially for 8-mer inhibitors, since they may be displaced during qPCR and thereby give an underrepresentation of miRNA inhibition. To demon- strate functional inhibition of a miRNA, it is important to show data from multiple assays, such as small RNA Northern blots, luciferase reporter assays, and target derepression, as shown in our study (2). Such data are lacking in the Thum et al. rebuttal, which makes comparison of the different chemistries impossible.

Thum et al. also state that we measured miR-21 inhibition on day 2 after dosing with Anti-21, when in fact we measured inhibition 3 weeks after dosing. At this time point, we observed inhibition of miR-21 in pressure-overloaded hearts at a level significantly below that of control mice. Thus, their approach for inhibition and/or measurement of miR-21 by their 8-mer inhibitors differs markedly from ours, since we observed robust miR-21 inhibition 3 weeks after injection, as demonstrated by multiple readouts (2). Using mismatched oligonucleotide controls is also important for interpreting miRNA inhibition studies in vivo, as described in our paper, rather than using PBS as a control, as reported by Thum et al. (1).
Finally, Thum et al. postulate that constitutive genetic deletion of miR-21 in mice may not reveal the functions of miR-21 in cardiac disease because of compensatory events that mask such functions. If such compensation occurs, it must be specific for the cardiac functions of miR-21, since miR-21 null mice are resistant to lung tumorigenesis (3), consistent with the documented pro-oncogenic functions of miR-21. To further address the possibility of genetic compensation, we have deleted a floxed miR-21 allele immediately prior to thoracic aortic constriction in mice using a ubiquitously expressed tamoxifen-regulated Cre transgene. These animals show cardiac hypertrophy and fibrosis comparable to that of their Cre-negative littermates. Genetic compensation therefore cannot account for the normal pathological cardiac remodeling response in miR-21 null mice. Moreover, functions of other miRNAs in heart disease can be revealed by genetic deletion in mice, as shown for miR-208 (4). Thus, while 22-mer oligonucleotide inhibitors against miR-21 are efficacious in inhib- 